\title{
Design and implementation of ergonomic performance measurement system at a steel plant in India
}

\author{
Pradip Kumar Ray, ${ }^{\mathrm{a},}$, V. K. Tewari ${ }^{\mathrm{b}}$ \\ ${ }^{a}$ Department of Industrial Engineering and Management, Indian Institute of Technology Kharagpur, Kharagpur \\ 721302, India, +913222 283742,pkr@vgsom.iitkgp.ernet.in \\ ${ }^{\mathrm{b}}$ Department of Agriculture and Food Engineering,, Indian Institute of Technology Kharagpur, Kharagpur 721 \\ 302, India, +91 3222 283152,vktfeb@agfe.iitkgp.ernet.in
}

\begin{abstract}
Management of Tata Steel, the largest steel making company of India in the private sector, felt the need to develop a framework to determine the levels of ergonomic performance at its different workplaces. The objectives of the study are manifold: to identify and characterize the ergonomic variables for a given worksystem with regard to work efficiency, operator safety, and working conditions, to design a comprehensive Ergonomic Performance Indicator (EPI) for quantitative determination of the ergonomic status and maturity of a given worksystem. The study team of IIT Kharagpur consists of three faculty members and the management of Tata Steel formed a team of eleven members for implementation of EPI model. In order to design and develop the EPI model with total participation and understanding of the concerned personnel of Tata Steel, a three-phase action plan for the project was prepared. The project consists of three phases: preparation and data collection, detailed structuring and validation of EPI model. Identification of ergonomic performance factors, development of interaction matrix, design of assessment tool, and testing and validation of assessment tool (EPI) in varied situations are the major steps in these phases. The case study discusses in detail the EPI model and its applications.
\end{abstract}

Keywords: base parameters, EPI Structure, worksystems, principal parameters, normalized total rating

\section{Introduction}

As a multidisciplinary subject the principles of ergonomics can be applied to the study and design of the components of any work system involving human(s) and machine(s) embedded in an environment, and as such its areas of application are not limited by a particular technology or by the scale of the system. In essence, application of these principles provide a standardized approach to the analysis of any worksystem with emphasis on consideration of interaction between human(s), machine(s), and environment. In this context, Tata Steel, Jamshedpur has been earnestly striving to apply the ergonomic principles, at its worksystems at different levels in order to ensure safety, health, convenience, and comfort of the personnel at their workplaces at an acceptable level of productivity and reliability of the overall system.

Application of ergonomic principles provides a standardized approach to the analysis of a worksystem with emphasis on evaluation of interaction between human(s), machine(s), and environment As a first step toward exploring the enormous potential and concepts of ergonomics at workplaces, the management of Tata Steel, the largest steel making company of India in the private sector, has taken steps to institutionalize the process of implementing ergonomics as a whole and felt the need to develop a framework to determine the levels of ergonomic performance at its different workplaces. As the idea of

*Pradip Kumar Ray. E-mail: pkray2010@gmail.com, pkr@vgsom.iitkgp.ernet.in. 
developing an ergonomic measurement system is quite unique, Tata Steel ventures into the design of a comprehensive approach for determining the ergonomic status of the worksystem with the help of a research team from IIT Kharagpur.

It has also been the opinion of the management that the factors of performance and/or operations where deficiencies or non-conformances occur should be identified and assessed on a regular basis to improve the performance, productivity, and reliability of any unit of analysis, and application of the concept of "remedial" ergonomics in many areas, operations, and factors of production may lead to substantial improvement in overall system performance. An assessment tool for determination of the status (level of ergonomic maturity) and level of ergonomic intervention to be employed in a given work system is all that is needed.

The IIT consulting team was requested to offer their expertise in the development of such an assessment tool for the Tata steel, Jamshedpur. As the idea of developing such an assessment tool is quite unique in the sense that for the first time, Tata Steel ventures into the design of a comprehensive approach for determining the ergonomic status of the worksystem, the IIT consulting team, discussed in detail the requirements of Tata Steel in this regard and agreed to offer its expertise.

\section{Objectives}

The objectives of the project are set as follows:

(i) to identify and characterize the ergonomic variables for a given worksystem with regard to work efficiency, operator safety, and working conditions,

(ii) to design a comprehensive Ergonomic Performance Indicator (EPI) for quantitative determination of the ergonomic status(in terms of design requirements and performance levelling) of a given worksystem or unit of analysis, and

(iii) to apply the EPI model to assess the degree of ergonomic maturity of a given worksystem or unit of analysis.

As decided by the management of the Tata Steel and agreed by the IIT consulting team, the functional scope of the project includes all types of worksystems and production units as identified by the concerned personnel of Tata Steel. Initially, a general framework of the assessment tool is needed before it is validated with respect to several representative worksystems of Tata Steel as decided by the management.

\section{Formation of project team}

The study team of IIT Kharagpur consists of three faculty members (with sufficient qualification, teaching and research experience in ergonomics and human factors) and the management of Tata Steel formed a team of eleven members who were primarily responsible for the work to be assigned by the IIT team and who could be approached for any kind of help and support required during the progress of the project work.

\section{Basic concepts}

In order to design an Ergonomic Performance Indicator (EPI) for determination of the ergonomic status of a given worksystem, modeling a general framework involving all relevant ergonomic factors is the essential first step. Assessing the degree of ergonomic maturity against each identified factor is the next important step.

The ergonomic factors to be considered in almost all situations or worksystems are related to four key aspects of worksystems, viz, human characteristics, physical workspace, physical environment, and organizational factors. While assessing the level of an ergonomic factor, three principal parameters need to be looked into, viz. work efficiency, operator safety, and working condition, each of which needs to be defined and interpreted in the widest possible sense during evaluation. The principal parameters are defined as follows:

(i) "Operator Safety" refers to either levels of outputs obtained per unit of time, or optimum time utilization, or minimum error rate in tasks, or efficiency in manual handling, or minimum energy expenditure rate by the person(s) concerned in a given worksystem.

(ii) "Work Safety" may be interpreted in terms of potential danger to health associated with the tasks, deteriorating fitness of the individual concerned, possibility of injuries and accidents, and hazards of any other kind.

(iii) "Working Condition" refers to the condition or environment in the workplace and its surroundings made available to the satisfaction or dissat- 
isfaction and comfort or discomfort of the person(s) concerned.

In essence, the performance of any worksystem is a reflection of the joint effect of performance of the individual components of the worksystem, viz. "human", "machine", and "environment", as illustrated in Figure 1.

It is reasonable to assume that for a worksystem to be capable of performing at its maximum level, each component must also contribute significantly and equally to the overall performance of the worksystem as a necessary condition for an acceptable worksystem. However, the central focus of ergonomics being the human(s) in a worksystem, the main consideration in the design of EPI is to measure and assess the state and the effect of human(s) in the worksystem, as a sufficient condition for sustained ergonomic performance. Hence, in order to assign values to the base parameters in EPI modelling, the effect on each component needs to be considered.

The three major components of "human", "machine", and "environment" have been assigned equal weightage while assessing the overall performance of a given worksystem. Against a base parameter, a three-level (L-I, L-II, and L-III) performance rating scale is recommended. For L-I, since the machine and the environment have the highest contribution and the human has the least contribution signifying the level of ergonomic maturity of the system, the total contribution of machine and environment is worked out to be 6.6 in a scale of $0-10$. For simplicity and convenience of use, a rounded off figure of 6 has been assigned to this level. Following the same logic, other two levels (L-II and L-III), depending on their ergonomic maturity, have been assigned a maximum score of 12 and 18 , respectively.

In the overall assessment of any type of worksystem (manufacturing or non-manufacturing, including service or office activities), various kinds of training programmes on safety may be initiated for the working personnel through safety campaign, and introduction of safe working methods. With regard to such conditions, it is proposed to assign an additional scale rating in a scale of $0-10$, on the basis of the intensiveness of safety programmes existing in the worksystem.

\section{Methodology}

The important steps of the methodology leading to the design and development of the EPI assessment tool are as follows:

(i) Identification of Ergonomic Factors

A general framework involving all relevant factors and sub-factors related to human characteristics, physical workspace, physical environment, and organizational factors is required to be developed including the procedures and norms to be followed for a given unit of analysis (worksystem). A structured data collection form, called EPI data record sheet (version 1) and coded as EPI-DRS-1, has been designed to elicit information and individual judgment from the Tata Steel team members on the ergonomic factors as identified by consultants, and to be included in the design of EPI.

(ii) Identification of Design and Performance Factors

A list of factors related to three specific aspects, viz. operator safety, work efficiency, and working condition including functional requirements, if any, is prepared and standardized at this stage. Wherever feasible and desired, these factors are objectively analyzed with respect to their current levels and/or implications; otherwise, they are subjectively assessed.

Initially, to identify all the possible ergonomic factors so as to have adequate breadth (coverage) and depth (intensiveness) in the EPI model, information on several ergonomic aspects in each factor needs to be considered. With an in-depth understanding of the prevailing situations and requirements of Tata Steel workplaces, several aspects representing depth of each factor, are identified by the consultants. 


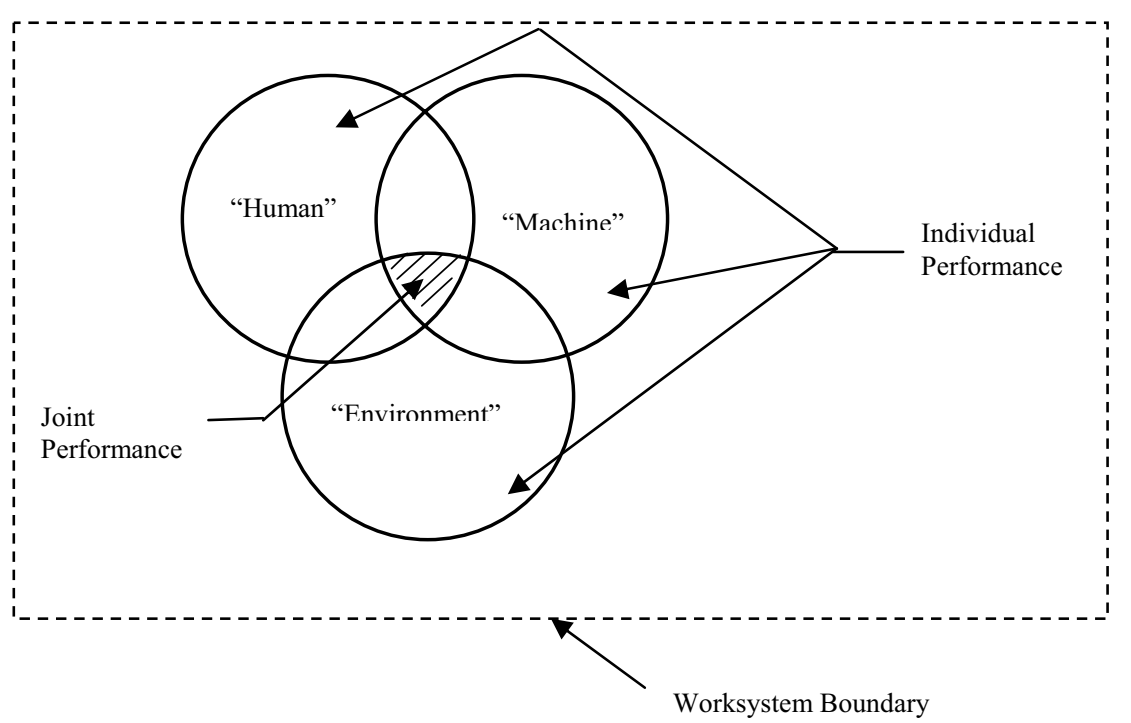

Figure 1: Overall Work Systems Performance: A Joint Effect of Individual Performance of Human, Machine, and Environment Components

(iii) Development of interaction matrix

At this stage, the interactions (strong or weak) between the ergonomic and design or performance factors to be ascertained for a given unit of analysis was prepared in order to limit the number of factors with which a given work system may be assessed to a reasonable size. The guidelines for the selection of appropriate number of factors is established. The rules for determining the relative weights (reflecting importance or criticality of a factor in the presence of other factors, or on its own) was specified at this stage.

(iv) Design of the assessment tool

On completion of the above three steps, a comprehensive framework for (1) determining the ergonomic performance of a worksystem (the basic EPI model), (2) identification of deficient area(s) in relation to ergonomic factor(s), and (3) setting the priority of improvement actions suggested, is established at this stage. Therefore, a structured methodology for measuring ergonomic performance of a worksystem, called EPI data record sheet (version 2) and coded as EPIDRS-2, is developed by the consultants for circulation and use among the Tata Steel personnel.

(v) Testing and validation of assessment tool (EPI) in varied situations and worksystems

The proposed tool is to be tested for its verification, validation, and applicability in a number of representative situations as specified and identified by the management of Tata Steel dur ing the course of study. Appropriate modification of the assessment tool is required to be made based on actual observations, review of opinion of Tata Steel personnel, and performance evaluation.

(vi) Data collection and analysis

The entire data collection and analysis was completed by the consultants through (i) meetings with the Tata Steel team members and other concerned personnel at regular and planned intervals, (ii) in-house preparation of required documents (EPI-DRS-1, EPI-DRS-2, and interaction matrix), (iii) visits to the selected and representative worksystems and departments at Tisco plant, (iv) verification and validation of the EPI model by hands-on exercises, and (v) discussion on the EPI model.

\section{EPI model: characteristic features}

The details of the EPI model designed and developed for Tata Steel, Jamshedpur, are described in three interrelated parts: Part-I, Part-II and Part-III. Part-I lists the ergonomic factors to be considered for EPI as well as the guidelines for quantitative assessment of base parameters in the design of EPI. Part-II refers to the tables required to be used to compute the EPI score of a given worksystem. There are five tables listed. The first table provides the definition of scale values for levelling and rating of the ergonomic variables considered; second table shows the details of the scale values under different conditions for assessment of visual environment; the third set of tables 
present the scale values under different conditions for assessment of thermal environment that requires evaluation of radiant temperature, air speed, and relative humidity, separately; the fourth table presents the details of the scale values under specified conditions and jobs for assessment of auditory environment. The data given in these tables are applicable in acclimatized conditions. The fifth table lists the possible EPI grading of a worksystem under consideration as well as status and actions for improvement. Part-III describes the systematic process of determining the EPI score of a given worksystem.

To understand how the EPI score is computed for a worksystem, the features and working of each part is to be known. The details of the features and working of each part is given below.

Part-I is designed to understand and quantitatively assess the importance of base parameters for a worksystem. In order to help define, assess, and quantify a parameter in the most logical and objective way, each ergonomic factor with its scale value is required to be defined for an objective assessment of base parameters. It is opined that the conditions as described in the guidelines are an exhaustive representation of different working conditions and systems at the present level of technology at Tata Steel.

It is recommended that the analyst studies the prevailing conditions against the following factors considered, with regard to key principal parameters viz., work efficiency, operator safety, and working condition, and matches with those given in the guidelines below. Against each factor, three specific rating scales representing acceptable to unacceptable levels are provided.

1. Pace or speed of work under the control of the operator

2. Adequacy of fatigue allowances for jobs

3. Workers away from their workplace during work

4. Occurrence of "human" errors

5. Frequency of lifting of weights

6. Force required to push or pull objects

7. Movements of human body

8. Assessment of visual environment in the workplace

9. Engineering Anthropometry

10. Work Posture

11. Assessment of thermal environment in the workplace

12. Workers complain about physical environment in their workplaces

13. Tasks resulting in excessive material wastes

14. Repetitive motions / frequent use of hand tools/ both hands and feet operating / same posture/ information overload/in sufficient time to sense and respond to signals/ physical fitness/

knowledge of training

15. Assessment of Auditory Environment

In Part II, each ergonomic variable or base parameter to be considered should be levelled and quantified, on a pre-defined scale, once the conditions are known to the analyst. Based on the consideration of the ergonomic effect of the specified conditions on the components of the worksystem, each condition is required to be quantified.

For determining the scale values of a specified ergonomic variable, a three-point scale is found to be applicable, feasible, and easily implementable. Each scale value, for which a level number is given, indicates a numeric ergonomic assessment score of a given situation or worksystem, based on the degree of maturity in the "human" component at the existing level of technology (defined in terms of "machine" and "environment" components). The definition of each level is provided.

While rating a given condition, it is quite probable that the degree of maturity of either 'machine' or 'environment' or both may also be medium or low. Under such conditions, the scale value is required to be suitably modified towards the lower side.

In Part-III, the steps to be followed for obtaining the EPI score of a worksystem are given. These steps are as follows:

Step-1: Select the principal parameter(s) relevant for the worksystem under consideration.

Step-2: Select the base parameter(s) influencing the identified principal parameter(s) in Step-1.

Step-3: Assess the situation against each base parameter considered (as described in Part-I Sec.5.1), and assign its scale rating (SR).

Step-4: Repeat Step-3 for all other base parameters selected.

Step-5: Compute the sum of scale ratings (SRs) obtained in Step-3 and Step-4.

Step-6: Assess the intensiveness of safety programmes adopted, and assign an appropriate safety awareness rating in a scale of (0-10).

Step-7: Compute the total ratings obtained in Step-5 and Step-6.

Step-8: Compute the normalized total rating (NTR) in a scale of (0-100). 


\section{EPI Grading}

The normalized total rating (NTR), as obtained in Step-8 above for a given worksystem may be graded belongigng to one of the five specific classes of worksystems given in Table 1. By using the EPI model, a worksystem may be of one of the five types: for Class-I or 'excellent worksystem', the prevailing work conditions need to be maintained, for Class-II or 'very good worksystem' refers to comparatively acceptable work condition and remedial steps wherever required may be initiated, for Class-III or 'good worksystem' refers to acceptable work condition with a great scope for improvement and a time-bound ergonomic intervention is required, for Class-IV or 'poor worksystem' refers to work condition not acceptable needing immediate ergonomic intervention, and for Class- $\mathrm{V}$ or 'very poor worksystem' means a work condition is rejected and large scale investment with intensive management involvement is required for improving the ergonomic performance.

\section{An application of EPI model}

The EPI model was verified and validated in several workplaces, such as blast furnace raw material division, merchant mill department for long products, LD2 department for flat products, equipment maintenance department, and power house\#4 of the steel plant. For illustration purpose, the method of computing EPI score, for two specific types of worksystems, viz., raw materials (RM) section of Blast Furnace (BF) and flat products department of LD\#2 plant of Tata Steel are explained below.

Application-I: for RM section of the Blast Furnace, the following factors are considered relevant for the purpose.

(i) Pace or speed of work under the control of the operator

(ii) Adequacy of fatigue allowances for jobs

(iii) Frequency of lifting of weights

(iv) Force required to push or pull objects

(v) Movements of human body

(vi) Assessment of visual environment in the workplace

(vii) Assessment of thermal environment in the workplace

(viii) Workers complain about physical environment in their workplaces (ix) Repetitive motions / frequent use of hand tools / both hands and feet operating / same posture / information overload / insufficient time to sense and respond to signals / physical fitness / knowledge of training

The other factors as listed in Part-I are not found to be relevant in this section.

The sum of scale ratings considering all the abovementioned factors is 81 (each of the factors is rated in a scale of 0-18). For such a system, the safety awareness is rated as 8 in (0-10) scale.

Hence, the Total Scale Ratings, TSR is $81+8=89$ For all the factors considered, the Maximum Scale Rating is $10 \times 18=180+10=190$

Hence, the Normalized Total Rating (NTR) is calculated as $89 / 190=0.47$

Referring to Table 1, it is found that a scale rating of 0.47 refers to a working condition that is not acceptable from ergonomic perspective and represents a poor worksystem.

Application-II: for flat products section of LD\#2 plant, the following factors are considered relevant for the purpose.

(i) Frequency of lifting of weights

(ii) Movements of human body

(iii) Assessment of visual environment in the workplace

(iv) Engineering Anthropometry

(v) Work Posture

(vi) Assessment of thermal environment in the workplace

(vii) Workers complain about physical environment in their workplaces

(viii) Repetitive motions / frequent use of hand tools / both hands and feet operating / same posture / information overload / insufficient time to sense and respond to signals / physical fitness / knowledge of training

(ix) Assessment of Auditory Environment

The other factors as listed in Part-I are not found to be relevant in this section.

The sum of scale ratings considering all the abovementioned factors is 87 (each of the factors is rated in a scale of $0-18$ ). For such a system, the safety awareness is rated as 8 in (0-10) scale.

Hence, the Total Scale Ratings, TSR $=87+8=95$

For all the factors considered, the Maximum Scale Rating is $18 \times 9=162+10=172$

The Normalized Total Rating (NTR) is calculated as $95 / 172=0.552$ 
Table 1: EPI Grading of Worksystems

\begin{tabular}{|l|l|l|l|}
\hline $\begin{array}{c}\text { Type } \\
\text { of } \\
\text { Worksytems }\end{array}$ & $\begin{array}{c}\text { Range } \\
\text { of } \\
\text { NTR }\end{array}$ & Grade & \multicolumn{1}{|c|}{ Remarks } \\
\hline Class-I & $85-100$ & Excellent & $\begin{array}{l}\text { Maintain the prevailing work con- } \\
\text { ditions. }\end{array}$ \\
\hline Class-II & $70-84$ & Very Good & $\begin{array}{l}\text { Comparatively acceptable work } \\
\text { condition; may initiate remedial } \\
\text { steps wherever required. }\end{array}$ \\
\hline Class-III & $50-69$ & Good & $\begin{array}{l}\text { Acceptable work condition with a } \\
\text { great scope for improvement; } \\
\text { time-bound ergonomic intervention } \\
\text { required. }\end{array}$ \\
\hline Class-IV & $45-49$ & Poor & $\begin{array}{l}\text { Work condition not acceptable; } \\
\text { needs immediate ergonomic inter- } \\
\text { vention. }\end{array}$ \\
\hline
\end{tabular}

Referring to Table 1, it is found that a scale rating of 0.552 refers to a working condition that is acceptable with enormous scope of improvement from ergonomic perspectives and represents a good worksystem.

\section{Lessons learned}

With the application of generic EPI model of several worksystems of Tata Steel, a number of observations regarding the usefulness of the model may be made:

i. The EPI model is applicable to all types of work systems

ii. With the use of EPI model on a continuous basis, there is a high probability that the persons at all levels of organization become aware of importance of ergonomic design for sustainable work system performance.

iii. As the model is factor specific, ability of the persons concerned to judge the ergonomic performance from several 'man- machine' perspectives is expected to improve in near future.

iv. The EPI model is cost-effective in the sense that it mainly suggests preventive measures for improving ergonomic performance and with the implementation of preventive measures, the effect of occupational risk factors get minimized.

\section{Conclusion}

The EPI model has been found to be very effective and a team of qualified ergonomics and human factors professionals from ergonomics and safety department of Tata Steel is responsible for computing EPI score of a given worksystem as and when required. The role of such professionals lies in assessing the present level of ergonomic performance and identifying the deficient areas (the list of factors where the scores are low) and the deficient areas where ergonomic interventions are desired.

It has been observed that such ergonomically deficient areas are regularly identified and in terms of ergonomic intervention projects are being undertaken by these professionals. In certain areas, external expertise in the form of hiring consultants is needed for undertaking such improvement initiatives. 